\title{
K-quantum Nonlinear Jaynes-Cummings Model in Two Trapped Ions *
}

\author{
Hao-Sheng Zeng ${ }^{1,2}$ \\ ${ }^{1}$ Laboratory of Magnetic Resonance and Atomic and Molecular \\ Physics, Wuhan Institute of Physics and Mathematics, Chinese Academy of \\ Science, Wuhan 430071, People's Republic of China \\ ${ }^{2}$ Department of Physics, Hunan Normal University, Hunan \\ 410081, People's Republic of China
}

\begin{abstract}
A k-quantum nonlinear Jaynes-Cummings model for two trapped ions interacting with laser beams resonant to k-th red side-band of center-of-mass mode, far from Lamb-Dicke regime, has been obtained. The exact analytic solution showed the existence of quantum collapses and revivals of the occupation of two atoms.
\end{abstract}

PACS numbers: 42.50. $\mathrm{Md}, 32.80 . \mathrm{Pj}$

*This work was supported by the National Natural Science Foundation of China (19734006 and 69873015) and Chinese Academy of Science 
In quantum optics, Jaynes-Cummings model(JCM) [1] has been regarded as a fundamental research model which can be exactly solved and predicts some interesting nonclassical effects both with respect to the atomic states and the boson mode, such as atomic revivals[2] and squeezing[3]. Experimental realization of JCM with dissipation has been demonstrated both in microwave and in optical domain. Observation of quantum revivals and nonclassical photon statistics with Rydberg atoms and microwave cavities has been reported [4], and in the optical regime vacuum Rabi splitting has been observed [5]. This experimental progress stimulated a manifold of further theoretical studies, for example of the quantum correlation between two interacting subsystems [6]. Moreover, related models have been rendered successively, such as nonlinearly coupled [7] and multiquantum JCMs [8], Raman-type [9], and three-level models [10].

Trapped ions interacting with laser beam is another fundamental system to exhibit, in appropriate limits, JCM dynamics. The quantized center-of-mass motion of the ion in the trap potential plays the role of the boson mode, which is coupled via the laser to the internal degrees of freedom. An analogy between an undamped trapped ion and the JCM has been noted by Blockley, Wall, and Risken [11], and by J. I. Cirac, et al., for damped trapped ion [12]. A study for nonlinear multiquantum JCM dynamics of a trapped ion is presented by W. Vogel and R. L. de Matos Filho [13]. A significant feature of trapped-ion JCM is that the effective coupling constant, which depends on Rabi frequency, could be readily adjusted in experiments to satisfy the strong coupling condition.

In recent experiments of trapped ions, entangled states up to four ions have been realized, and some applications, such as the proof of Bell's inequality and decoherence-free quantum memory, have been confirmed[14]. These improvements offered some advantageous conditions for studying properties of multi-ion JCM.

In this paper we will demonstrate that a system comprising two trapped ions interacting with a laser beam resonant to k-th red side-band of center-of-mass mode, far from LambDicke regime, shows a nonlinear JCM dynamics. Collapses and revivals can be observed in this system. These properties of internal states for two trapped ions are useful for quantum 
computation. Because quantum manipulation involves at lest two-bit operations, and only we know the properties of internal state of ions very clearly, can we manipulate them well and truly. In addition, the model for this system may be realized in present experimental conditions[14], which will result in directly the tests of the predictions from this paper.

Consider two ions trapped in a linear trap which are strongly bounded in the $y$ and $z$ directions but weakly bounded in a harmonic potential in $x$ direction.. Assuming that the two ions are illuminated simultaneously with a dispersive beam resonant with k-th red side-band of center-of-mass mode, so that the effects from the relative motional mode can be neglected because of the very large off-resonant reason[14,15]. The Hamiltonian for this system is

$$
\begin{gathered}
H=H_{0}+H_{\text {int }} \\
H_{0}=\nu\left(a^{+} a+1 / 2\right)+\omega \sum_{i=1}^{2} \sigma_{i z} / 2 \\
H_{\text {int }}=\sum_{i=1}^{2} \frac{\Omega}{2}\left\{\sigma_{+i} e^{i \eta\left(a+a^{+}\right)} e^{-i(\omega-k \nu) t}+H . C .\right\}
\end{gathered}
$$

where $\nu$ and $a^{+}(a)$ are the frequency and ladder operators of the center-of-mass mode. $\omega$ is the energy difference between the ground state $|0\rangle$ and the long-lived metastable excited state $|1\rangle$ of the ion. For simplicity we further assume that the Rabi frequency and Lamb-Dicke parameter for the two ions are same. Transforming the Hamiltonian (3) to the interaction picture with respect to $H_{0}$ and making use of rotating wave approximation, we have,

$$
H_{\text {int }}=\Omega J_{+} f\left(a^{+} a\right) a^{k}+H . C .
$$

with

$$
f\left(a^{+} a\right)=e^{-\eta^{2} / 2} \sum_{n=0}^{\infty} \frac{(i \eta)^{2 n+k}}{n !(n+k) !}\left(a^{+}\right)^{n} a^{n}
$$

where $J_{ \pm}=J_{x} \pm i J_{y}$ with $J_{\alpha}=\frac{1}{2}\left(\sigma_{1 \alpha}+\sigma_{2 \alpha}\right)(\alpha=x, y, z)$ being the three components of total spin operator of the two ions. 
E.q.(4) is namely the so-called k-quantum nonlinear JCM Hamiltonian. When any one of the two ions is excited, the quantum number of $J_{z}$ component of the total spin operator increases by one, and in the meantime, the quantum number of center-of-mass mode decreases by $k$.

Following the method of [16], we can get the exact solution of Hamiltonian (4) after a tedious deduction,

$$
\begin{gathered}
U_{11}=\frac{1}{A^{2}+B^{2}}\left[A^{2} \cos \left(\sqrt{A^{2}+B^{2}} t\right)+B^{2}\right] \\
U_{00}=\cos \left(\sqrt{A^{2}+B^{2}} t\right) \\
U_{-1-1}=\frac{1}{A^{2}+B^{2}}\left[B^{2} \cos \left(\sqrt{A^{2}+B^{2}} t\right)+A^{2}\right] \\
U_{10}=-U_{01}^{*}=\frac{-i^{k+1} A}{\sqrt{A^{2}+B^{2}}} \sin \left(\sqrt{A^{2}+B^{2}} t\right) \\
U_{1-1}=U_{-11}=(-1)^{k+1} \frac{A B}{A^{2}+B^{2}}\left[1-\cos \left(\sqrt{A^{2}+B^{2}} t\right)\right] \\
U_{0-1}=-U_{-10}^{*}=\frac{-i^{k+1} B}{\sqrt{A^{2}+B^{2}}} \sin \left(\sqrt{A^{2}+B^{2}} t\right)
\end{gathered}
$$

where $A=\sqrt{2} \Omega e^{-\eta^{2} / 2} \eta^{k} \sqrt{\frac{(n-2 k) !}{(n-k) !}} L_{(n-2 k)}^{k}\left(\eta^{2}\right)$ and $B=\sqrt{2} \Omega e^{-\eta^{2} / 2} \eta^{k} \sqrt{\frac{(n-k) !}{n !}} L_{(n-k)}^{k}\left(\eta^{2}\right)$ with $L_{n}^{k}(x)=\sum_{m=0}^{n}(-1)^{m}\left(\begin{array}{c}n+k \\ n-m\end{array}\right) \frac{x^{m}}{m !}$.

In order to study the phenomena of collapses and revivals of the two ions, imitating traditional process, we assume that initially the internal states of the two ions are both in the ground states and the external motional state of center-of-mass mode in a coherent state $|\alpha\rangle$. Thus the whole initial state of the system is,

$$
\rho(0)=\sum_{m^{\prime}, m^{\prime \prime}=0}^{\infty} q\left(m^{\prime}\right) q^{*}\left(m^{\prime \prime}\right)\left|-1 m^{\prime}\right\rangle\left\langle-1 m^{\prime \prime}\right|
$$


with $q(m)=\exp \left(-\frac{1}{2}|\alpha|^{2}\right) \frac{\alpha^{m}}{\sqrt{m} !}$ are the coefficients for the coherent state expanding in the number state basis.

The density operator of the internal state of this system can be obtained by tracing over the vibrational motion of center-of-mass mode. With above equations, we can get the time evolution of diagonal elements of the density-matrix for the two-ion system,

$$
\begin{gathered}
\rho_{11}(t)=\sum_{n=2}^{\infty} \frac{A^{2} B^{2}}{\left(A^{2}+B^{2}\right)^{2}}\left[1-\cos \left(\sqrt{A^{2}+B^{2}} t\right)\right]^{2} p(n) \\
\rho_{00}(t)=\sum_{n=2}^{\infty} \frac{B^{2}}{A^{2}+B^{2}}\left[\sin \left(\sqrt{A^{2}+B^{2}} t\right)\right]^{2} p(n)+[\sin B(1) t]^{2} p(1) \\
\rho_{-1-1}(t)=\sum_{n=2}^{\infty} \frac{1}{\left[A^{2}+B^{2}\right]}\left[A^{2}+B^{2} \cos \left(\sqrt{A^{2}+B^{2}} t\right)\right]^{2} p(n)+[\cos B(1) t]^{2} p(1)+p(0)
\end{gathered}
$$

with $B(1)=\sqrt{2} \Omega e^{-\eta^{2} / 2} \eta$ and

$$
p(n)=e^{-|\alpha|^{2}} \frac{|\alpha|^{2 n}}{n !}
$$

is the probability distribution of phonon number for coherent state of center-of-mass mode.

According to E.q.(13)-(15), we plotted the exact time-evolution of diagonal elements of density-matrix as in Fig.1, which describes the time-evolution of occupation probabilities of electronic energy-levels. we assumed that initially the internal states of the two ions are both in ground, and the vibration motion of center-of-mass mode is in a coherent state with average phonon number $|\alpha|^{2}=10$. The typical parameters are $\eta=0.1$ and $\Omega=2 \pi \times 500$ $\mathrm{kHz}$ [14]. From these curves, we can see clearly the collapses and revivals for the electronic state occupation of the two trapped ions. It is worth while to point out that, in this system, collapses and revivals increase with the increase of average phonon number, but decrease with the increase of Lamb-Dicke. In order to observe collapses and revivals in the case of large Lamb-Dicke parameters, we must enhance average phonon number. As a comparison, we plotted the similar figure with the same Rabi frequency and parameters $\eta=0.2,|\alpha|^{2}=50$ and $\eta=0.4,|\alpha|^{2}=80$ in Fig. 2. Obviously the collapses and revivals in Fig.2 (b) is very obscure, because of the very large Lamb-Dicke parameter. 
Collapses and revivals in two-quantum resonance are very similar to the case of onequantum resonance. The occupation distribution of internal energy-level for different LambDicke parameters and different average phonon number for this case are shown in Fig.3(the Rabi frequency is same as above.).

In conclusion, we showed that the behavior of two trapped ions, interacting with a laser beam resonant to k-th red sand-band of center-of-mass mode, can be described by a nonlinear JCM mode under appropriate limits. An exact analytic solution for this type of JCM was presented and the results indicated obvious collapses and revivals. We expect the similar behaviors for more than two ions, and also expect new features of the nonclassical statistics of the vibrational mode and of other effects known from the standards JCMs.

Fig.1. The occupations $\rho_{11}(t), \rho_{00}(t), \rho_{-1-1}(t)$ of electronic state of the two ions for onequantum resonance are given as functions of time with parameters: $\eta=0.1, \Omega=2 \pi \times 500$ $\mathrm{kHz}$. Initially the motional state of center-of-mass mode be in a coherent state with average phonon number $|\alpha|^{2}=10$ and the two ions be in ground state.

Fig.2. The time-evolution of the occupations $\rho_{11}(t)$ of excited electronic state of the two ions for one-quantum resonance. (a) $\eta=0.2,|\alpha|^{2}=50$; (b) $\eta=0.4|\alpha|^{2}=80$.

Fig.3. The occupations $\rho_{11}(t)$ of excited electronic state of the two ions for two-quantum resonance are given as functions of time with parameters: (a) $\eta=0.1,|\alpha|^{2}=20$; (b) $\eta=0.2,|\alpha|^{2}=50 ;$ (c) $\eta=0.4,|\alpha|^{2}=80$. 


\section{REFERENCES}

[1] E. T. Janes and F. W. Cummings, Proc. IEEE 51, 89 (1963).

[2] J. H. Eberly, N. B. Narozhny and J. J. Sanchez-Mondragon, Phys. Rev. Lett. 44, 1323 (1980); M. Fleischhauer and W. Schleich, Phys. Rev. A 47, 4258 (1993).

[3] P. Meystre and M. S. Zubairy, Phys. Lett. 89A, 390 (1982).

[4] G. Rempe, H. Walther and N. Klein, Phys. Rev. Lett. 58, 353 (1987); G. Rempe, F. Schmidtkaler and H. Walther, ibid. 64, 2783 (1990).

[5] M. G. Raizen, et al., Phys. Rev. Lett. 63, 240 (1989); Y. Zhu et al., ibid. 64, 2499 (1990); G. Rempe et al., ibid. 67, 1727 (1991); R. J. Thompson, G. Rempe and H. J. Kimble, ibid. 68, 1132 (1992).

[6] S. J. D. Phoenix and P. L. Knight, Phys. Rev. A44, 6023 (1991); S. M. Barnett and S. J. D. Phoenix, ibid. 40, 2404 (1989); J. Gea-Banacloche, Phys. Rev. Lett. 65, 3385 (1990).

[7] B. Buck and C. V. Sukumar, Phys. Lett. 81A, 132 (1981); C. V. Sukumar and B. Buck, ibid. 83A, 211 (1981).

[8] W. Vogel, D.-G. Welsch and L. Leine, J. Opt. Soc. Am. B4, 1633 (1987); W. Vogel and D.-G. Welsch, Phys. Rev. A 40, 7113 (1989)

[9] P. L. Knight, Phys. Scr. T12, 51 (1986).

[10] H.-I. Yoo and J. H. Eberly, Phys. Rep. 118, 239 (1985).

[11] C. A. Blockley, D. F. Wall and H. Risken, Europhys. Lett. 17, 509 (1992).

[12] J. I. Cirac, R. Blatt, A. S. Parkins and P. Zoller, Phys. Rev. Lett. 70, 762 (1993).

[13] W. Vogel and R. L. de Matos Filho, Phys. Rev. A 52, 4214 (1995).

[14] C. A. Sackett, et al. Nature 404, 256 (2000); M. A. Rowe, et al. ibid. 409,791 (2001); 
D. Kielpinski, et al. Science 291, 1013 (2001).

[15] A. S $\phi$ rensen and K. M $\phi$ lmer, Phys. Rev. Lett. 82, 1971(1999); K. M $\phi$ lmer and A. S $\phi$ rensen, ibid. 82, 1835 (1999).

[16] Hao-Sheng Zeng, et al. quant-ph/0106020. 